\title{
Infection control in dentistry: compliance with guidelines needs more work
}

\author{
How well do dental team members follow infection control guidelines?
}

\section{Gordon BL, Burke FJT, Bagg J, Marlborough HS, McHugh ES. Systematic review of adherence to infection control guidelines in dentistry. J Dent 2001; 29:509-516}

Data sources Data sources were MEDLINE, EMbase, BIDS Science Citation Index and Social Sciences Citation Index, the Cochrane Library, NHS EED (NHS Economic Evaluation Database), SIGLE (System for Information on Grey Literature in Europe), British Dental Association Library, reference lists of identified studies and hand searches of the International Dental Journal, Community Dentistry and Oral Epidemiology and Journal of the American Dental Association.

Study selection Randomised controlled trials (RCT), controlled clinical trials, controlled before-and-after studies, interrupted time series, observational studies, surveys and reports were selected. A wide range of outcome measures, both observed and self-reported, were considered, for example, glove use, mask use, wearing of protective clothing and eye wear and vaccination against hepatitis B virus.

Data extraction and synthesis Two reviewers independently selected studies and the quality was assessed using a checklist. Disagreements were resolved by discussion. Due to the degree of heterogeneity, a qualitative synthesis was performed.

Results Only 71 studies met the inclusion criteria and their overall quality was poor. The results were summarised under several headings: knowledge and attitudes, personal protective equipment, immunisation, sterilisation and disinfection, waste disposal, and occupationally acquired injuries. They revealed substantial improvements in compliance in some areas of infection control such as glove wearing, but other aspects, such as the effective management of needlestick injuries, remains problematic.

Conclusions More rigorously designed studies are needed to accurately assess dental team members' adherence to infection control guidelines.

\section{Commentary}

As the authors point out in their review, the increased profile of blood-borne viruses such as human immunodeficiency and hepatitis $B$ and $C$ viruses has resulted in increased scrutiny of the infection control procedures applied in dentistry. The review may be best-described as diffuse, in that it covers a field of dentistry rather that the more focused single questions one associates with a Cochrane review. As a result of this it suffers to a degree, although it is also hampered by the poor quality of the primary research in this area. Whereas the methodological approach looks good, it is disappointing that the inclusion/exclusion criteria are not more clearly defined. This review does, however, provide a useful summary of the best available information at present.

The review identifies variations in dentists' knowledge of and adherence to infection-control procedures both within developed and developing countries. Circumstances are clearly different in different countries and some measures will involve financial constraints, but dentists' knowledge should be the same and the gaps need to be addressed.

Hepatitis B vaccination has been available for many years and the review finds that uptake has increased but that postexposure follow-up is often overlooked. Occupational health support for dental practices in relation to this could be improved and some moves are being made towards that in the UK. The role of the dental nurse or surgery assistant in infection control is central and their compliance with guidelines is described as not adequate. This may be related to level of training which varies greatly, although no great detail is presented in relation to this in the review.

The emergence of prion diseases will place increasing demands on practitioners in their compliance with infection-control procedures. The review provides a useful assessment of where we are now in this regard, and how much needs to be done in the future. It also indicates that we need much better designed research to assess dental team compliance with infection guidelines and procedures.

\section{Practice point}

- Compliance with infection control procedures has improved but more work needs to be done to address the important gaps that do exist.

\author{
Derek Richards \\ Centre for Evidence-based Dentistry, Oxford, UK
}

Evidence-Based Dentistry (2003) 4, 33.

doi:10.1038/sj.ebd.6400179 